\title{
Covid-19 e pratiche postnatali: una moderna parabola
}

\author{
RICCARDO DAVANZO', FABIO MOSCA ${ }^{2}$ \\ "IRCCS Materno-Infantile "Burlo Garofolo", Trieste \\ ${ }^{2}$ Neonatologia e Terapia Intensiva Neonatale, Fondazione IRCCS Ca' Granda Ospedale Maggiore Policlinico, Milano
}

I contenuti delle linee guida più recenti, comprese quelle della Società Italiana di Neonatologia, sull'assistenza dei nati da madre Covid-19, sono noti. Vale la pena però approfondirne in maniera ordinata le motivazioni, affinché gli operatori sanitari possano applicarle in modo diffuso e omogeneo.

$\mathrm{L}$ a pandemia da Covid-19 ha determinato dei cambiamenti organizzativi improvvisi nel Sistema Sanitario e in particolare nell'area delle cure alla maternità. Sono emerse nuove priorità operative e in molti contesti ospedalieri sono state prese decisioni essenzialmente finalizzate a evitare a ogni costo il rischio infettivo, senza preoccuparsi troppo se i provvedimenti presi, declinati in nuovi protocolli e nuove pratiche, avrebbero potuto compromettere la qualità dell'assistenza personalizzata a mamma e bambino. Si è così deciso di ridurre i contatti personali per visite, test e trattamenti nel percorso nascita, si è esclusa la presenza del partner durante travaglio e parto, si sono separati nel post partum la madre Covid-19-positiva dal neonato nel timore di contagio del coronavirus, le indicazioni al cesareo si sono allargate, sono stati poste restrizioni e/o proibizioni all'allattamento al seno ${ }^{1}$.

In un clima di incertezza senza precedenti, "navigating the uncharted" dice un Editoriale di Fauci e coll. sul New England Journal of Medicine $e^{2}$, a luglio 2020 erano giustamente già disponibili una ventina di linee guida, giudicate di modesta qualità metodologica ${ }^{3}$. Quello che colpisce però è il fatto che mostrassero una grande intervariabilità a fonte delle limitate, ma comuni,

POSTNATAL PRACTICES AND THE COVID-19 MOTHER

(Medico e Bambino 2020;39:65 1-655)

Key words

Guidelines, Breastfeeding, Covid-19, Skin-to-skin contact, Rooming-in

\section{Summary}

Early in the Covid-19 pandemic maternity hospital practices were jeopardized by guidelines produced by scientific societies to reduce the risk of mother to child transmission of the SARS-CoV-2 infection to a minimum. Subsequent research has documented that skin-toskin contact in the delivery room, feeding directly to the breast and rooming-in can be considered safe, if infection control measures are concurrently applied. Professionals should not forget the proven benefits of breastfeeding and the importance of early motherbaby relationship whenever taking decisions that might hinder these practices.

conoscenze scientifiche in quel momento disponibili su cui queste linee guida avrebbero dovuto basarsi. Questo fenomeno non può essere spiegato se non con un'attitudine professionale differente da parte di chi, all'interno delle Società scientifiche, aveva il mandato di procedere all'estensione delle stesse raccomandazioni.

\section{PRATICHE POSTPARTUM E TRASMISSIONE ORIZZONTALE DEL CORONAVIRUS}

La trasmissione verticale dell'infezione da SARS-CoV-2 è possibile, ma molto rara e quindi nel valutare il rischio di trasmissione materno-infantile vanno prese piuttosto in considerazio- ne le modalità attualmente note di trasmissione orizzontale ${ }^{4}$. Queste sono essenzialmente tre. Citandole per ordine di importanza: 1 . mediante le goccioline respiratorie (droplet), 2. per contatto diretto e 3. mediante l'aerosol. 1. Le cosiddette goccioline hanno un diametro maggiore ai 5 micron, cadono dove vengono espirate (entro 1 metro) e spiegano la raccomandazione di tenere una distanza di almeno 1 metro (meglio se 2 metri) dall'individuo infetto o potenzialmente tale e di usare le mascherine chirurgiche.

2. Il contatto diretto avviene mediante oggetti o feci o la superficie cutanea contaminati, ed è noto che, quantomeno su plastica e acciaio, il virus 


\begin{tabular}{|l|c|}
\hline \multicolumn{2}{|c|}{ CONCENTRAZIONE DI SARS-CoV-2 SU CAMPIONI DI ARIA } \\
AMBIENTALE, ESPRESSA IN COPIE DI RNA VIRALE SU LTRO
\end{tabular}

Tabella I

possa sopravvivere fino a 72 ore $^{5}$. $\mathrm{Si}$ ipotizza che le mani contagiate portino a un'auto-inoculazione, peraltro mal documentabile, attraverso le mucose dell'occhio, bocca e naso. La possibilità di questo contagio diretto è alla base della raccomandazione di pulire periodicamente le superfici ambientali con acqua e sapone e/o con i disinfettanti.

3. Le micro-goccioline (con diametro $<5$ micron) sono capaci di disperdersi nell'aria ambiente anche a distanza superiore al metro. Qualora veicolino il coronavirus, questo si troverà a concentrazioni tanto più elevate quanto più vicino al paziente infetto e quanto inferiore sia il ricambio di aria (Tabella I) $)^{4}$. Questa via di trasmissione del coronavirus è stata nel corso della pandemia progressivamente valorizzata ed è alla base del suggerimento di curare il paziente Covid-19-positivo in stanze a pressione negativa oppure che possano prevedere l'apertura delle finestre per il ricambio dell'aria, a conferma del valore dei saggi vecchi consigli prodigati da mamme e nonne.

\section{ROOMING-IN DI PUERPERA E NEONATO}

Nel momento in cui si indichi la separazione della madre Covid-19-positiva dal suo neonato si rende ovviamente impossibile non solo il rooming-in (definita come gestione congiunta di madre e neonato nella stessa stanza) ma, in considerazione dell'interdipendenza delle pratiche postnatali, anche il contatto pelle a pelle e l'allattamento diretto al seno.

I benefici del rooming-in, caldamente promosso dall'Iniziativa Ospedale Amico del Bambino dell'OMS/UNI$\mathrm{CEF}^{6}$, sono ben provati scientificamente. In particolare questa modalità di gestione idealmente $24 / 24$ ore, giorno e notte, non solo facilità il bonding e l'avvio dell'allattamento, ma consente alla madre, specie se alla prima esperienza di maternità, di fare una pratica supervisionata dallo staff ospedaliero. Inoltre il rooming-in permette una più facile e precoce identificazione di un eventuale disagio psicologico materno. Le conseguenze della sostituzione del rooming-in con la gestione separata di madre e bambino sono molteplici e a più livelli e non vanno assolutamente sottovalutate. Aumentano preoccupazione e stress materni. Al neonato non viene garantita la protezione da un'eventuale esposizione al coronavirus da parte del personale ospedaliero che lo accudisce durante la permanenza in ospedale e dopo la dimissione dall'ospedale l'esposizione da parte di caregiver o da contatto con individui positivi. Siccome la mancanza del roomingin è frequente causa di non allattamento, il bambino potrà trovarsi nei mesi successivi maggiormente a rischio di malattie infettive diverse dal coronavirus gravate nei bambini vulnerabili da peggiori complicazioni ${ }^{7}$. Anche la famiglia può soffrire degli effetti negativi della separazione, specialmente quan- do manchino le risorse materiali, umane e logistiche per garantire a domicilio il completamento della quarantena di 14 giorni $^{8}$. Infine il mancato rooming-in pesa in maniera non sostenibile su Servizi sanitari già scarsi di personale e sopraffatti dal carico assistenziale da pandemia. Insomma la separazione di routine non rappresenta la giusta risposta?.

\section{IL CONTATTO PELLE A PELLE}

Esistono barriere alla sua implementazione poste sostanzialmente dagli operatori sanitari della Sala Parto, che hanno storicamente addotto il problema della mancanza di tempo per sostenerlo o più recentemente del rischio di collasso neonatale ${ }^{10}$. Il timore che una mamma Covid-19-positiva possa passare l'infezione al proprio bambino durante la pratica del pelle a pelle è solo l'ultima barriera in ordine temporale. È documentato che il coronavirus presente eventualmente sulla pelle sopravvive 9 ore, a fronte delle 1,8 ore del virus dell'influenza $A^{11}$. Questo però non è comunque sufficiente per rendere plausibile la trasmissione al neonato del SARS-CoV-2 eventualmente presente sulla pelle materna contaminata dalle secrezioni respiratorie della madre stessa. Si dovrebbe ipotizzare che il neonato disposto sul torace-addome materni inali il bio-aerosol di liquidi presenti sulla pelle materna o che il coronavirus sia capace di trasmettersi per via enterale dopo che il neonato abbia leccato o succhiato la pelle materna. Queste possibilità non sono documentate (bio-aerosol) oppure non sono giudicate possibili (trasmissione orofecale). Un altro dubbio è stato sollevato relativamente alla responsabilità infettante del secreto vaginale delle partorienti Covid-19-positive. Nello studio di Fenizia e coll. su 30 donne al parto ${ }^{12}$ il secreto vaginale è risultato positivo in un caso di grave Covid-19 gravidico con parto pretermine. Infine anche il liquido amniotico, altro fluido che può riscontrarsi sulla pelle della puerpera, è solitamente negativo per SARS-CoV2 , a meno di casi molto rari di trasmissione verticale. 
Nel momento in cui viene proibita (o continua a essere proibita) la pratica del pelle a pelle, se ne ignorano di fatto i benefici quali l'adeguata termoregolazione del neonato, un suo più rapido adattamento alla nuova vita, la riduzione del pianto, l'avvio precoce e la promozione dell'allattamento e del bon$d i n g^{13}$. Insomma, il pelle a pelle non va inteso come una semplice fugace e bella esperienza a valenza esclusivamente psicologica, ma come una pratica capace di recare salute, non ultimo per la possibilità di modificare assieme al latte materno il microbiota intestinale del neonato.

\section{ALLATTAMENTO E LATTE MATERNO}

Anche i molteplici e documentati benefici dell'allattamento al seno ${ }^{14}$ andrebbero tenuti a mente ogni qualvolta si prendano decisioni mediche di carattere clinico o organizzativo, che possano in maniera diretta o indiretta interferire con il suo successo e/o la sua reputazione. Questo purtroppo non è avvenuto, quantomeno inizialmente, in occasione dell'attuale pandemia. Il latte materno è stato sospettato di essere responsabile della trasmissione verticale del coronavirus da madre a bambino, pur in assenza di dati scientifici a sostegno di questa ipotesi, semplicemente sulla base del fatto che altri virus (HIV o virus dell'immunodeficienza umana, HTLV o virus T-linfotropico dell'uomo, citomegalovirus, Ebola) lo fanno. È mancata una saggia valutazione bilanciata da un lato dei rischi (ipotetici e non documentati) dell'allattamento da parte di una donna Covid-19-positiva, dall'altra della perdita dei benefici (ben documentati e quindi certi) conseguente al non allattamento al seno.

Allo stato attuale delle conoscenze, il latte materno prodotto da una donna Covid-19-positiva può raramente risultare positivo all'RNA del SARS-CoV-2, senza però che a questa positività corrisponda una competenza a replicarsi da parte del virus (colture negative) ${ }^{15}$. Il latte materno è inoltre dotato di un'attività antivirale per effetto della lattoferrina ${ }^{16}$, ma soprattutto per la presenza di anticorpi IgA ad azione neutralizzante diretti contro il Receptor Binding Domain (RBD) del coronavirus ${ }^{17}$.

In sintesi, il latte materno non va ritenuto come un liquido biologico capace di infettare il neonato ${ }^{18}$ né va ritardato il suo avvio, che dovrebbe avvenire già in Sala Parto nelle primissime ore di vita.

\section{QUALI RISCHI PER IL FIGLIO DI DONNA CON COVID-19?}

Rischio di trasmissione materno-infantile

La percentuale di positività per SARS-CoV-2 dei figli di gravide Covid19-positive risulti nel complesso bassa: $4,5 \%$ per l'osservatorio nazionale del Regno Unito ${ }^{19}, 2 \%$ negli USA ${ }^{20}, 2,5 \%$ secondo il Registro Nazionale Covid-19 della Società Italiana di Neonatologia INNSIN-Covid-19 ${ }^{21}$. Anche la revisione sistematica della letteratura effettuata da Walker e coll. su 666 neonati figli di gravide Covid-19-positive indica una percentuale complessiva di trasmissione del $4 \%^{22}$; maggiore dopo un parto mediante taglio cesareo $(5,3 \%)$ rispetto a uno vaginale $(2,7 \%)$ e maggiore nei neonati separati dalle loro madri e nutriti con formula rispetto a quelli del rooming-in (13\% vs 3,7\%). Questi studi sono stati condotti su neonati prevalentemente gestiti in un regime di rooming-in dalle loro madri, seguendo strategie di controllo della trasmissione dell'infezione: uso di mascherine, pulizia delle mani e spesso anche del seno prima delle poppate, pulizia/disinfezione ambientale, distanziamento di almeno 2 metri, allattamento al seno e/o con latte materno spremuto, anche se non sempre in maniera esclusiva, limitazione o proibizione delle visite nel post partum (a volte compreso il padre del bambino).

A conferma di quanto sopra affermato vanno citate alcune altre esperienze.

Uno studio spagnolo su 22 figli di donne Covid-19-positive ha focalizzato l'esperienza in Sala Parto: il 54\% dei neonati veniva allattato al seno e il $59 \%$ sperimentava la pratica del pelle a pelle. Nei primi 2 mesi di vita sono rimasti asintomatici e per il $77 \%$ allattati al seno $0^{23}$.
In uno studio condotto su 3 ospedali di New York, un gruppo di neonati di madre Covid-19-positiva, che nell' $83 \%$ dei casi era rimasto nella stessa stanza della madre, è stato sottoposto a controlli nel primo mese di vita, mostrando negatività del test per SARS-CoV-2 e buone condizioni di salute $^{24}$.

Infine, in un studio condotto nel New Jersey, solo 1 su 49 neonati è risultato transitoriamente positivo al SARS-CoV-2 a 24 ore di vita, negativizzandosi a 48 ore $^{25}$. Questi bambini erano stati gestiti in un regime di rooming-in e allattati al seno. Un teleconsulto nei primi 14 giorni di vita aveva evidenziato che stavano bene.

In sintesi allo stato attuale delle conoscenze né la pratica del pelle a pelle né il rooming-in né l'allattamento diretto al seno possono essere ritenuti significativi fattori di rischio di trasmissione, sempre che si rispettino le norme di protezione e controllo della trasmissione delle infezioni respiratorie. Ferrazzi e coll., riferendo l'esperienza di 12 ospedali del Nord Italia, riportano come i casi di positività per SARS-CoV2 neonatale possano dipendere dall'identificazione ritardata al parto delle puerpere Covid-19-positive con conseguente gap temporale nell'uso della mascherina e delle precauzioni anticontagio nel postpartum ${ }^{26}$.

\section{Gravità dell'infezione in età neonatale}

Infezione precoce. Nonostante la preoccupazione avanzata da alcuni sull'eventuale necessità di assistenza intensiva per i bambini SARS-CoV-2 positivi nati da madri Covid-19-positive, vi è attualmente una sufficiente esperienza clinica per affermare che questi bambini sono il più delle volte asintomatici o con sintomi lievi e/o aspecifici.

In caso di prematurità. Il tasso di prematurità nelle gravide Covid-19-positive è pari a 2-3 volte quello della popolazione femminile in epoca pre-Covid-19 $9^{19,27,28}$. In Italia l'Istituto Superiore di Sanità riporta che il confronto fra questi valori è di $19,2 \%$ versus $7 \%{ }^{29}$. Il messaggio che l'infezione neonatale 
precoce sia silente o lieve non può però valere, almeno in via di principio, per il sottogruppo numericamente non trascurabile dei neonati SARS-CoV-2 pretermine. $\grave{E}$ atteso che questi bambini possano andare incontro a tutte le complicazioni, anche gravi, della prematurità. Resta comunque ancora da stabilire in che misura le condizioni cliniche di un neonato pretermine SARS-CoV-2 positivo dipendano dalla prematurità e quanto piuttosto da una concomitante maggiore espressività del Covid-19 nel pretermine.

Outcome a lungo termine. Al momento le infezioni neonatali alla nascita sembrano avere un esito favorevole, senza casi documentati fino a questo momento di exitus, sempre che non vi

\section{MESSAGGI CHIAVE}

I neonati infetti da SARS-CoV-2 solitamente sono asintomatici o paucisintomatici, a meno che non coesista uno stato di significativa prematurità che può condizionare la prognosi complessiva.

- Il contatto pelle a pelle in Sala Parto, I'allattamento diretto al seno materno e il rooming-in sono da intendersi come sicuri quando si seguano le norme di controllo di trasmissione dell'infezione (mascherine, distanziamento, lavaggio mani e superfici ambientali). Ogni scelta alternativa (isolamento della madre dal bambino, distacco immediato in Sala Parto, alimentazione con formula) rappresenta una soluzione imperfetta che ha implicazioni peggiorative.

$\square$ Le indicazioni della Società Italiana di Neonatologia emesse fin dall'inizio della pandemia hanno mantenuto la loro validità dopo 8 mesi, alla luce delle nuove evidenze scientifiche.

$\square$ La American Academy of Pediatrics ha modificato le sue iniziali indicazioni sull'assistenza al neonato figlio di madre Covid-19-positiva, allineandosi alle linee guida europee.

$\square$ Un insegnamento da questa pandemia è che gli operatori sanitari dovrebbero essere molto cauti ogniqualvolta linee guida, protocolli e schemi organizzativi mettano a rischio la relazione madre-bambino e l'allattamento. si associ una patologia rilevante come la grave prematurità.

Infezioni tardive. I neonati che si infettano con SARS-CoV-2 in ambiente intra-familiare e che vengono ospedalizzati sono in genere più gravi dei neonati con infezione precoce, manifestando solitamente febbre, sintomi respiratori, difficoltà alimentari, come risulta anche dall'analisi preliminare del Registro Italiano INNSIN-Covid-1921. Non abbiamo evidenze chiare, ma le infezioni nel corso del primo mese di vita potrebbero essere simili in fase acuta a quelle di altri noti virus respiratori, capaci di dare a seconda dei casi il semplice raffreddore, ma anche più raramente quadri più gravi o gravissimi di bronchiolite o di polmonite.

Resta da chiarire, mediante studi di follow-up dei neonati con infezioni tardive e sintomatiche da SARS-CoV-2, quale possa essere la funzionalità respiratoria e il neuro-sviluppo a medio e lungo termine.

\section{QUALE LEZIONE ABBIAMO APPRESO?}

Le indicazioni ad interim della Società Italiana di Neonatologia (SIN) uscite in 3 versioni successive fra il 28 febbraio e il 10 maggio $2020^{30,31}$ hanno supportato il ritardato clampaggio del funicolo e la pratica del pelle a pelle, il rooming-in e l'allattamento per il neonato figlio di donna Covid-19-positiva. Il principio al quale queste indicazioni ad interim si ispiravano era quello di proteggere, per quanto possibile, la relazione madre-bambino, avendola valutata come prioritaria rispetto a una possibile infezione neonatale precoce da SARS-CoV2 clinicamente lieve o più spesso muta.

La SIN, a febbraio 2020, seguiva le indicazioni dell'OMS ${ }^{32}$ contrapponendosi cosi alle raccomandazioni provenienti dalla $\operatorname{Cin}^{33} \mathrm{e}$ a quelle inizialmente stilate dall'American Academy of Pediatrics $(\mathrm{AAP})^{34}$. Le evidenze scientifiche in seguito disponibili hanno confermato l'appropriatezza della posizione dalla SIN, inducendo la stessa AAP a rielaborare a luglio 2020 le proprie raccomandazioni sull'esempio europeo ${ }^{35}$.

\section{CONCLUSIONI}

L'elaborazione delle linee guida riguardanti le pratiche postnatali in corso di pandemia da Covid-19, così variabili fra loro, rappresenta quasi una moderna parabola che sottolinea come ogniqualvolta ci troviamo in presenza di limitate evidenze scientifiche ci sia il rischio (e forse la tentazione) di penalizzare le pratiche postnatali.

Nelle linee guida, così come nelle scelte organizzative e nelle quotidiane decisioni cliniche, è invece necessario sviluppare un'attitudine maggiormente attenta all'importanza della relazione madre-bambino, ai benefici dell'allattamento e in definitiva alla loro reputazione.

\section{Indirizzo per corrispondenza:}

Riccardo Davanzo

e-mail: riccardo.davanzo@gmail.com

\section{Bibliografia}

1. Renfrew MJ, Cheyne H, Craig J, et al. Sustaining quality midwifery care in a pandemic and beyond. Midwifery 2020;88:102759.

2. Fauci AS, H Clifford Lane H, Redfield RR. Covid-19 - Navigating the uncharted. N Engl J Med 2020;382(13):1268-9.

3. Yeo KT, Oei JL, De Luca D, et al. Review of guidelines and recommendations from 17 countries highlights the challenges that clinicians face caring for neonates born to mothers with Covid-19. Acta Paediatr 2020 Jul 27 [Epub ahead of print].

4. Morawska L, Tang JW, Bahnfleth W, et al. How can airborne transmission of Covid-19 indoors be minimised? Environ Int 2020;142: 105832 .

5. van Doremalen N, Bushmaker T, Morris $\mathrm{DH}$, et al. Aerosol and surface stability of SARS-CoV-2 as compared with SARS-CoV-1. N Engl J Med 2020;382(16):1564-7.

6. WHO, UNICEF. Protecting, promoting and supporting breastfeeding in facilities providing maternity and newborn services: the revised Baby-Friendly Hospital Initiative. Geneva, 2018.

7. Stuebe A. Should infants be separated from mothers with Covid-19? First, do no harm. Breastfeed Med 2020;15(5):351-2.

8. Arora KS, Mauch JT, Gibson KS. Labor and delivery visitor policies during the Covid19 pandemic: balancing risks and benefits. JAMA 2020;323(24):2468-9.

9. Tomori C, Gribble K, Palmquist AEL, Ververs MT, Gross MS. When separation is not the answer: Breastfeeding mothers and infants affected by Covid-19. Matern Child Nutr 2020;16(4):e13033. 
10. Davanzo R, Merewood A, Manzoni P. Skin-to-skin contact at birth in the Covid-19 Era: in need of help! Am J Perinatol 2020;37 (S 02):S1-S4

11. Hirose R, Ikegaya H, Naito Y, et al. Survival of SARS-CoV-2 and influenza virus on the human skin: Importance of hand hygiene in Covid-19. Clin Infect Dis 2020:ciaa1517.

12. Fenizia C, Biasin M, Cetin I, et al. Analysis of SARS-CoV-2 vertical transmission during pregnancy. Nat Commun 2020;11(1): 5128 .

13. Moore ER, Bergman N, Anderson GC, Medley N. Early skin-to-skin contact for mothers and their healthy newborn infants. Cochrane Database Syst Rev 2016;11: CD003519.

14. Section on Breastfeeding. Breastfeeding and the use of human milk. Pediatrics 2012; 129(3):e827-41.

15. Chambers C, Krogstad P, Bertrand K, et al. Evaluation for SARS-CoV-2 in breast milk from 18 infected women. JAMA 2020;324 (13):1347-8.

16. Peroni DG, Fanos V. Lactoferrin is an important factor when breastfeeding and Covid19 are considered. Acta Paediatr 2020: 10.1111/apa.15417 [Epub ahead of print].

17. Pace RM, Williams JE, Järvinen KM, et al. Covid-19 and human milk: SARS-CoV-2, antibodies, and neutralizing capacity. MedRxiv 2020:2020.09.16.20196071.

18. Shenker NS, Wesolowska A, van Goudoever JB, Nangia S, Klotz D. Undermining breastfeeding will not alleviate the Covid-19 pandemic. Lancet 2020;396(10257):1064-5. 19. Knight M, Bunch K, Vousden N, et al.; UK Obstetric Surveillance System SARSCoV-2 Infection in Pregnancy Collaborative Group. Characteristics and outcomes of pregnant women admitted to hospital with con- firmed SARS-CoV-2 infection in UK: national population based cohort study. BMJ 2020; 369:m2107.

20. Dumitriu D, Emeruwa UN, Hanft E, et al. Outcomes of neonates born to mothers with severe acute respiratory syndrome coronavirus 2 infection at a large medical Center in New York City. JAMA Pediatr 2020:e204298 [Epub ahead of print]

21. Gizzi C. INNSIN COVID-19. Registro Covid-19 del Network della Società Italiana di Neonatologia (SIN). Relazione al Congresso Nazionale della SIN, Venezia, 10 ottobre 2020. 22. Walker KF, O'Donoghue K, Grace N, et al. Maternal transmission of SARS-CoV-2 to the neonate, and possible routes for such transmission: a systematic review and critical analysis. BJOG 2020;127(11):1324-36.

23. Pereira A, Cruz-Melguizo S, Adrien M, et al. Breastfeeding mothers with Covid-19 infection: a case series. Int Breastfeed J 2020; 15(1):69.

24. Salvatore CM, Han JY, Acker KP, et al. Neonatal management and outcomes during the Covid-19 pandemic: an observation cohort study. Lancet Child Adolesc Health 2020;4(10):721-7.

25. Kest H, Kaushik A, Skroce L, Bogusz J, Datta-Bhutada S. Rooming-in for well term infants born to asymptomatic mothers with Covid-19. J Pediatric Infect Dis Soc 2020: piaa120 [Epub ahead of print].

26. Ferrazzi E, Frigerio L, Savasi V, et al. Vaginal delivery in SARS-CoV-2-infected pregnant women in Northern Italy: a retrospective analysis. BJOG 2020;127(9):1116-21. 27. Martínez-Perez O, Vouga M, Melguizo $\mathrm{SC}$, et al. Association between mode of delivery among pregnant women with Covid-19 and maternal and neonatal outcomes in Spain. JAMA 2020;324(3):296-9.
28. Remaeus K, Savchenko J, Brismar Wendel S, Brusell Gidlöf S, Graner S. Characteristics and short-term obstetric outcomes in a case series of 67 women tested positive for SARS-CoV-2 in Stockholm, Sweden. Acta Obstet Gynecol Scand. 2020;99(12):1626-31.

29. Maraschini A, Corsi E, Salvatore MA, Donati S; ItOSS Covid-19 Working Group. Coronavirus and birth in Italy: results of a national population-based cohort study. Ann Ist Super Sanità 2020;56(3):378-89.

30. Davanzo R, Moro G, Sandri F, Agosti M, Moretti C, Mosca F. Breastfeeding and coronavirus disease-2019. Ad interim indications of the Italian Society of Neonatology endorsed by the Union of European Neonatal \& Perinatal Societies. Matern Child Nutr 2020: e13010.

31. Società Italiana di Neonatologia (SIN). Allattamento e gestione del neonato in corso di pandemia da SARS-COV-2. Indicazioni ad interim. Versione 3 del 10 maggio 2020

32. WHO. Clinical management of Severe Acute Respiratory Infections (SARI) when Covid-19 disease is suspected. Interim Guidance. Geneva, 13th March 2020.

33. Wang L, Shi Y, Xiao T, et al. Working Committee on Perinatal and Neonatal Management for the Prevention and Control of the 2019 Novel Coronavirus Infection. Chinese expert consensus on the perinatal and neonatal management for the prevention and control of the 2019 novel coronavirus infection (first edition). Ann Transl Med 2020;8(3):47. 34. Puopolo KM, Hudak ML, Kimberlin DW, Cummings J. Initial guidance: management of infants born to mothers with Covid-19. American Academy of Pediatrics Apr 02, 2020.

35. Sulaski Wyckloff A. Rooming-in, with precautions, now OK in revised AAP newborn guidance. AAP News, July 22, 2020 . 